\title{
Far-field Approximations to the Derivatives of the Green's Function for the Ffowcs Williams and Hawkings Equation
}

\section{Zhiteng Zhou}

Chinese Academy of Sciences Institute of Mechanics

\section{Zhengyu Zang}

Chinese Academy of Sciences Institute of Mechanics

\section{Hongping Wang}

Chinese Academy of Sciences Institute of Mechanics

Shizhao Wang ( $\square$ wangsz@Inm.imech.ac.cn )

Chinese Academy of Sciences Institute of Mechanics

\section{Research Article}

Keywords: Ffowcs Williams and Hawkings integral, Green's function, high-order derivatives, far-field approximation, frequency-domain formulation, multipole expansion

Posted Date: November 30th, 2021

DOI: https://doi.org/10.21203/rs.3.rs-1079048/v1

License: (c) (i) This work is licensed under a Creative Commons Attribution 4.0 International License. Read Full License

Version of Record: A version of this preprint was published at Advances in Aerodynamics on March 16th, 2022. See the published version at https://doi.org/10.1186/s42774-022-00109-x. 


\section{RESEARCH}

\section{Far-field approximations to the derivatives of the Green's function for the Ffowcs Williams and Hawkings equation}

Zhiteng Zhou ${ }^{1,2}$, Zhenyu Zang ${ }^{1,2}$, Hongping Wang ${ }^{1}$ and Shizhao Wang ${ }^{1,2 *}$

\footnotetext{
${ }^{*}$ Correspondence:

wangsz@lnm.imech.ac.cn

${ }^{1}$ Institute of Mechanics, Chinese

Academy of Sciences, Beijing,

China

Full list of author information is

available at the end of the article
}

\begin{abstract}
The surface correction to the quadrupole source term of the Ffowcs Williams and Hawkings integral in the frequency domain suffers from the computation of high-order derivatives of the Green's function. The far-field approximations to the derivatives of the Green's function have been used without derivation and verification in the previous work. In this work, we provide the detailed derivations of the far-field approximations to the derivatives of the Green's function. The binomial expansions for the derivatives of the Green's function and the far-field condition are employed during the derivations to circumvent the difficulties in computing the high-order derivatives. The approximations to the derivatives of the Green's function are systemically verified by using the benchmark two dimensional convecting vortex and the co-rotating vortex pair. In addition, we provide the derivations of the approximations to the multiple integrals of the Green's function by using the far-field approximations to the derivatives.
\end{abstract}

Keywords: Ffowcs Williams and Hawkings integral; Green's function; high-order derivatives; far-field approximation; frequency-domain formulation; multipole expansion

\section{Introduction}

The Ffowcs Williams and Hawkings (FW-H) equation [1] is among the most popular acoustic analogy method to predict sound generated by unsteady flows. It has been successfully used to predict the sound generated by flows from the jet engines $[2$, $3]$, propellers [4] ,hydrofoils [5], high-speed trains $[6,7]$ the ducted tail rotor [8] and biomimetic asymmetric bar [9]. The FW-H equation is a non-homogeneous wave equation which extends the Lighthill's acoustic analogy to problems with arbitrary moving permeable/non-permeable boundaries. The general form of the FW-H equation with a uniform convective flow can be given as follows [10],

$$
\begin{array}{r}
\left(\frac{\partial^{2}}{\partial t^{2}}+M_{i} M_{j} \frac{\partial^{2}}{\partial x_{i} \partial x_{j}}+2 M_{i} \frac{\partial^{2}}{\partial x_{i} \partial t}-\frac{\partial^{2}}{\partial x_{i} \partial x_{i}}\right)\left(H(f) p^{\prime}\right)= \\
\frac{\partial^{2}}{\partial x_{i} \partial x_{j}}\left(T_{i j} H(f)\right)-\frac{\partial}{\partial x_{i}}\left(F_{i} \delta(f)\right)+\frac{\partial}{\partial t}(Q \delta(f))
\end{array}
$$

where $p^{\prime}=p-p_{o}$ is the pressure fluctuation. $p$ is the pressure and $p_{o}$ is the ambient pressure at the far-field pressure. We use the superscript $(\cdot)^{\prime}$ to denote the fluctuation, subscript $(\cdot)_{o}$ to denote the far-field variable. $t$ is time. $x_{i}(i=1,2,3)$ are 
the independent variables in the Cartesian coordinate system defined by $o-x_{1} x_{2} x_{3}$. $M_{i}=U_{i} / c_{o}$ is the freestream Mach number along the $o-x_{i}$ axis where $U_{i}$ is component of the freestream velocity. $c_{o}$ is the sound speed. $f$ is the signed distance function that defines the permeable/non-permeable boundary by $f=0 . H(f)$ is the Heaviside function and $\delta(f)$ is the Dirac delta function. $T_{i j}=\rho u_{i} u_{j}+P_{i j}-c_{o}^{2} \rho / \delta_{i j}$ is the Lighthill stress tensor, where $\rho$ is the density, $u_{i}$ is the component of fluid velocity along the $o-x_{i}$ axis. $P_{i j}=\left(p-p_{o}\right) \delta_{i j}-\tau_{i j}$ is the compressive stress tensor, where $\rho l=\rho-\rho_{o}$ is the density fluctuation, $\rho_{o}$ is the far-field density in the ambient medium, $\delta_{i j}$ is the Kronecker delta function and $\tau_{i j}$ is the viscous stress tensor. $F_{i}=\left(P_{i j}+\rho\left(u_{i}-U_{i}\right)\left(u_{j}+U_{j}\right)+\rho_{o} U_{i} U_{j}\right) n_{j}$, where $n_{j}$ is the normal vector of the permeable/non-permeable surface defined by $f=0 . Q=\left(\rho\left(u_{i}+U_{i}\right)-\rho_{o} U_{i}\right) n_{i}$. The three terms on the right-hand-side of the FW-H equation $\frac{\partial}{\partial t}(Q \delta(f)),-\frac{\partial}{\partial x_{i}}\left(F_{i} \delta(f)\right)$, and $\frac{\partial^{2}}{\partial x_{i} \partial x_{j}}\left(T_{i j} H(f)\right)$ are usually termed as monopole source, dipole source, and quadrupole source, respectively.

The solution to the FW-H equation can be expressed as surface integrals of monople source and dipole source and a volume integral of the quadrupole source. Hereinafter, we refer these integrals as monople source term, dipole source term, and quadrupole source term, respectively. The quadrupole source term is usually ignored under the assumption that the monopole source term and dipole source term dominate the far-field sound in the low Mach number flows [11]. However, recent researches show that ignoring the quadrupole source term may result in significant spurious sound even at relatively low Mach numbers $[12,13,14]$. Different methods have been proposed to eliminate or suppress the spurious sound [15, 16, 17, 18, 14].

One of the most widely used method to eliminate the spurious sound is the surface correction integral proposed by Wang et al. [16]. The idea of the surface correction integral is to account for the contribution of the quadrupole sources outside the integral domain, because the spurious sound is generated by the eddies crossing the boundary of the integral domain. With the frozen eddy assumption, the contribution of the quadrupole sources outside the integral domain can be modeled by using the quadrupole source flux on the boundary of the integral domain. Then the surface correction integral can be constructed based on the quadrupole source flux accounting for the contributions of the quadrupole sources outside the integral domain. The spurious sound has been eliminated or dramatically reduced by the surface correction integral in computing the sound generated by flows over airfoils [16]. However, the surface correction integral requires that the eddies leave the boundary of the integration domain at a nearly constant speed. To improve the surface correction integral proposed by Wang et al. [16], different methods have been proposed by accounting for the non-constant convective velocity at the boundary of the integral domain $[18,13]$. Most of the surface correction integrals are developed within the time-domain framework.

Lockard and Casper [17] firstly proposed the surface correction integral for the FW-H equation within the frequency domain. The derivation of the surface correction integral for the frequency-domain method involves repeating integration by parts. Thus, the surface correction integral reported by Lockard and Casper [17] 
consists of a series of surface integrals as follows,

$$
I_{Q}(\mathbf{x} ; \omega) \approx-\sum_{l=1}^{n} \int_{f=0}\left(\frac{-\mathrm{i} U_{1}}{\omega}\right)^{l} T_{i j}(\mathbf{y}, \omega) \frac{\partial^{l-1}}{\partial y_{1}^{l-1}}\left(\frac{\partial^{2} G(\mathbf{x} ; \mathbf{y})}{\partial y_{i} \partial y_{j}}\right) d S
$$

where $f=0$ is the boundary of the volume integral of quadrupole source in $\mathrm{FW}-\mathrm{H}$ equation (hereinafter referred to as FW-H integral surface). $\mathrm{i}=\sqrt{-1}$ is the unit of the imaginary number. Without loss of generality, we set the convective velocity of the quadrupole sources along the $o-x_{1}$ direction and use $U_{1}$ to denote the convective velocity. $G(\mathbf{x} ; \mathbf{y})$ is the Green's function, where $\mathbf{x}$ and $\mathbf{y}$ are the observer and source position, respectively. $\omega$ is the frequency. The surface correction integral for the frequency-domain method (Eq. (2)) works reasonably well in the test cases of convecting vortex when the convective Mach number of the flow $M_{a}=U_{1} / c_{o}$ is lower than 0.5 . The problems with the surface correction integral for the frequencydomain method (Eq. (2)) are that (1) the series of surface integrals on the righthand-side of Eq.(2) might be divergent when the convective Mach number is higher than $0.5,(2)$ the computation of the high-order derivatives of the Green's function is quite complicated and nontrivial [17]. The divegence of Eq. (2) is caused by the phenomenon that the convective velocity is larger than the phase velocity of a perturbation propagating through the convective flows. The far-field approximations to the derivatives of the Green's function have also been utilized to fix the second problem of the surface correction integral. However, these far-field approximations to the derivatives of the Green's function have been used without derivation and verification [19].

The aim of this work is to give the detailed derivations and verification of the approximations to the derivatives of the Green's function. The derivations of the approximations are based on the binomial expansion of the derivatives of the Green's function and the far-field condition. These approximations to the derivatives are verified in details by computing the far-field acoustic pressure from the quadrupole source term generated by the two-dimensional convecting vortex and the co-rotating vortex pair. In addition, the approximations to the integrals of the Green's function are derived by using the approximations to the derivatives of the Green's function.

The remainder of the paper is organized as follows. We will give the derivations of the approximations to the derivatives of the Green's function for $2 \mathrm{D}$ and 3D flows in Section 2. The verifivations and applications of the approximations to the derivatives of the Green's function are reported in Section 3. Finally, the conclusions are drawn in Section 4.

\section{Far-field approximations to the derivatives of the Green's function}

We investigate the FW-H equation in the form of convective wave equation in the frequency domain in accordance with the work of Lockard et al. [10, 17]. The Green's function for the convective wave equation in the frequency domain is simply referred to as the Green's function hereinafter when there is no ambiguity. We directly give the expressions of the far-field approximations to the derivatives of the Green's function in subsection 2.1. Then, we provide the detailed derivations of the approximations in subsection 2.2 . 


\subsection{Expressions of the far-field approximations to the derivatives of the Green's function}

The $(l+2)$ th-order derivative of the Green's function with respect to the variables $y_{q}, y_{i}$ and $y_{j}$ can be approximated at the far field as follows,

$$
\frac{\partial^{l}}{\partial y_{q}^{l}}\left(\frac{\partial^{2} G(\mathbf{x} ; \mathbf{y})}{\partial y_{i} \partial y_{j}}\right) \approx\left(\frac{\partial \varphi(\mathbf{x} ; \mathbf{y})}{\partial y_{q}}\right)^{l} \frac{\partial^{2} G(\mathbf{x} ; \mathbf{y})}{\partial y_{i} \partial y_{j}}
$$

where $G$ is the Green's function, $\varphi$ is the phase function of the Green's function.

For the two-dimensional (2D) flows, we have $G \approx G_{2 D}$ and $\varphi=\varphi_{2 D}$ in the far field with

$$
\begin{aligned}
& G_{2 D}=\frac{\mathrm{i}}{4 \beta}\left(\frac{2 \beta^{2}}{\pi k R}\right)^{\frac{1}{2}} \exp ^{\varphi_{2 D}}, \\
& \varphi_{2 D}(\mathbf{x} ; \mathbf{y})=\mathrm{i}\left[\frac{M k\left(x_{1}-y_{1}\right)}{\beta^{2}}+\frac{\pi}{4}-\frac{k}{\beta^{2}} R\right],
\end{aligned}
$$

where the observer and source locations are denoted by $\mathbf{x}$ and $\mathbf{y}$, respectively. $k=$ $\omega / c_{o}$ is the wavenumber. $\beta=\sqrt{1-M^{2}}$ is the Prantle-Glauert factor. $M$ is the Mach number of the freestream flow. The distance $R$ is computed by,

$$
R=R_{2 D}=\sqrt{\left(x_{1}-y_{1}\right)^{2}+\beta^{2}\left(x_{2}-y_{2}\right)^{2}} .
$$

It is noted that the 2D Green's function $G_{2 D}$ given in Eq. (4) is the asymtotic Green's function for 2D flows.

For the three-dimensional (3D) flows, we have $G=G_{3 D}$ and $\varphi=\varphi_{3 D}$ with

$$
\begin{aligned}
& G_{3 D}=-\frac{1}{4 \pi R} \exp ^{\varphi_{3 D}}, \\
& \varphi_{3 D}(\mathbf{x} ; \mathbf{y})=-\mathrm{i} k \frac{\left(R-M\left(x_{1}-y_{1}\right)\right)}{\beta^{2}},
\end{aligned}
$$

where the distance $R$ is computed by,

$$
R=R_{3 D}=\sqrt{\left(x_{1}-y_{1}\right)^{2}+\beta^{2}\left(x_{2}-y_{2}\right)^{2}+\beta^{2}\left(x_{3}-y_{3}\right)^{2}} .
$$

By using the approximations to the derivatives to the Green's function (Eq. (3)), the surface correction integral (Eq. (2)) proposed by Lockard and Casper [17] can be approximated by

$$
I_{Q}(\mathbf{x} ; \omega) \approx-\sum_{l=1}^{n} \int_{f=0}\left(\frac{U_{1}}{i \omega}\right)^{l} T_{i j}(\mathbf{y}, \omega)\left(\frac{\partial \varphi(\mathbf{x} ; \mathbf{y})}{\partial y_{1}}\right)^{l-1} \frac{\partial^{2} G(\mathbf{x} ; \mathbf{y})}{\partial y_{i} \partial y_{j}} d S .
$$

The high-order derivatives of the Green's function $\frac{\partial^{l-1}}{\partial y_{1}^{l-1}}\left(\frac{\partial^{2} G(\mathbf{x} ; \mathbf{y})}{\partial y_{i} \partial y_{j}}\right)$ in Eq. (2) are approximated by $\left(\frac{\partial \varphi(\mathbf{x} ; \mathbf{y})}{\partial y_{1}}\right)^{l-1} \frac{\partial^{2} G(\mathbf{x} ; \mathbf{y})}{\partial y_{i} \partial y_{j}}$ in Eq. (10). Therefore, the difficulties in computing the high-order derivatives of the Green's function are circumvented. 
2.2 Derivatives of the approximations to the derivatives of the Green's function at the far field

The approximations in Eq. (3) can be derivated separately by setting $q=$ $1, \cdots, N D$, where $N D=2$ for two-dimensional flows and $N D=3$ for threedimensional flows. We report the detailed derivation of the approximations to the derivatives of the Green's function when $q=1 \mathrm{in} \mathrm{Eq.} \mathrm{(3).} \mathrm{The} \mathrm{same} \mathrm{derivations} \mathrm{can}$ be obtained following the same way for $q=2$ and $q=3$. The subscript $q=1$ in Eq. (3) indicates that the $l$ th-order derivative of $\frac{\partial^{2} G(\mathbf{x} ; \mathbf{y})}{\partial y_{i} \partial y_{j}}$ is taken along the direction of $o-y_{1}$ axis. When the subscript $q=1$, Eq. (3) can be expressed as

$$
\frac{\partial^{l}}{\partial y_{1}^{l}}\left(\frac{\partial^{2} G(\mathbf{x} ; \mathbf{y})}{\partial y_{i} \partial y_{j}}\right) \approx\left(\frac{\partial \varphi(\mathbf{x} ; \mathbf{y})}{\partial y_{1}}\right)^{l} \frac{\partial^{2} G(\mathbf{x} ; \mathbf{y})}{\partial y_{i} \partial y_{j}}
$$

We re-write the left-hand-side of Eq. (11) as

$$
\frac{\partial^{l}}{\partial y_{1}^{l}}\left(\frac{\partial^{2} G(\mathbf{x} ; \mathbf{y})}{\partial y_{i} \partial y_{j}}\right)=\frac{\partial^{2}}{\partial y_{i} \partial y_{j}}\left(\frac{\partial^{l} G(\mathbf{x} ; \mathbf{y})}{\partial y_{1}^{l}}\right)
$$

For the two-dimensional flows with $G=G_{2 D}$, the $l$ th-order of derivative of the Green's function with respect to the variable $y_{1}$ can be expressed with the binomial expansion as follows

$$
\frac{\partial^{l} G_{2 D}(\mathbf{x} ; \mathbf{y})}{\partial y_{1}^{l}}=\frac{i}{4 \beta}\left(\frac{2 \beta^{2}}{\pi k}\right)^{1 / 2} \sum_{k_{1}=0}^{l} C_{l}^{k_{1}} \frac{\partial^{k_{1}} \exp ^{\varphi(\mathbf{x} ; \mathbf{y})}}{\partial y_{1}^{k_{1}}} \frac{\partial^{l-k_{1}}\left(R^{-1 / 2}\right)}{\partial y_{1}^{l-k_{1}}}
$$

where $C_{l}^{k_{1}}$ is the binomial coefficient.

We consider the the far-field sound generated by the compact source flows. Without loss of generality, we set the origin of the reference frame near the compact source flows. For the far-field sound at the observation position $\mathbf{x}$ generated by the sources at the position $\mathbf{y}$, we have $|\mathbf{x}|>>|\mathbf{y}|$. The $k_{1}$ th-order of derivative to the distance $R$ given by Eqs. (8) and (9) can be approximated by

$$
\frac{\partial^{k_{1}} R}{\partial y_{1}^{k_{1}}} \approx O\left(\frac{1}{R^{k_{1}-1}}\right) \frac{\partial R}{\partial y_{1}}
$$

Similarly, we have

$$
\frac{\partial^{k_{1}}\left(R^{-1 / 2}\right)}{\partial y_{1}^{k_{1}}} \approx O\left(\frac{1}{R^{k_{1}-1}}\right) \frac{\partial R^{-1 / 2}}{\partial y_{1}},
$$

By using Eq. (14), the $k_{1}$ th-order derivative of $\exp ^{\varphi(\mathbf{x} ; \mathbf{y})}$ can be approximated by

$$
\frac{\partial^{k_{1}} \exp ^{\varphi(\mathbf{x} ; \mathbf{y})}}{\partial y_{1}^{k_{1}}} \approx\left(\frac{\partial \varphi(\mathbf{x} ; \mathbf{y})}{\partial y_{1}}\right)^{k_{1}} \exp ^{\varphi(\mathbf{x} ; \mathbf{y})}\left(k_{1} \geq 1\right)
$$


Thus, the binomial expansion of Eq. (4) can be expressed as,

$$
\begin{aligned}
\frac{\partial^{l} G_{2 D}(\mathbf{x} ; \mathbf{y})}{\partial y_{1}^{l}} & =\frac{i}{4 \beta}\left(\frac{2 \beta^{2}}{\pi k}\right)^{1 / 2} \sum_{k_{1}=0}^{l} C_{l}^{k_{1}} \frac{\partial^{k_{1}} \exp ^{\varphi(\mathbf{x} ; \mathbf{y})}}{\partial y_{1}^{k_{1}}} \frac{\partial^{l-k_{1}}\left(R^{-1 / 2}\right)}{\partial y_{1}^{l-k_{1}}} \\
& \approx \frac{i}{4 \beta}\left(\frac{2 \beta^{2}}{\pi k}\right)^{1 / 2} \sum_{k_{1}=0}^{l} C_{l}^{k_{1}}\left(\frac{\partial \varphi(\mathbf{x} ; \mathbf{y})}{\partial y_{1}}\right)^{k_{1}} \exp ^{\varphi(\mathbf{x} ; \mathbf{y})} \frac{\partial^{l-k_{1}}\left(R^{-1 / 2}\right)}{\partial y_{1}^{l-k_{1}}}
\end{aligned}
$$

Further, the derivatives of $R^{-1 / 2}$ terms on the right-hand-side of Eq. (17) can be approximated according to Eq. (15). Thus, we get the far-field approximations to the $(l+2)$ th-order derivatives of the Green's function for the two-dimensional flows with $q=1$ as follows,

$$
\frac{\partial^{l}}{\partial y_{1}^{l}}\left(\frac{\partial^{2} G_{2 D}(\mathbf{x} ; \mathbf{y})}{\partial y_{i} \partial y_{j}}\right) \approx\left(\frac{\partial \varphi_{2 D}(\mathbf{x} ; \mathbf{y})}{\partial y_{1}}\right)^{l} \frac{\partial^{2} G_{2 D}(\mathbf{x} ; \mathbf{y})}{\partial y_{i} \partial y_{j}}
$$

For three-dimensional flows with $G=G_{3 D}$, the $l$ th-order derivative of the Green's function with respect to the variable $y_{1}$ can be expressed with the binomial expression as follows

$$
\frac{\partial^{l} G_{3 D}(\mathbf{x} ; \mathbf{y})}{\partial y_{1}^{l}}=\frac{-1}{4 \pi} \sum_{k_{1}=0}^{l} C_{l}^{k_{1}} \frac{\partial^{k_{1}} \exp ^{\varphi(\mathbf{x} ; \mathbf{y})}}{\partial y_{1}^{k_{1}}} \frac{\partial^{l-k_{1}}\left(R^{-1}\right)}{\partial y_{1}^{l-k_{1}}}
$$

For the far-field sound at the observation position $\mathbf{x}$ generated by the sources at the position $\mathbf{y}$, we have $|\mathbf{x}|>>|\mathbf{y}|$. The approximated $k_{1}$ th-order of derivative to the distance $R$ given by Eq. (14) is then employed to give the three-dimensional formulation of Eq. (16). Therefore, the binomial expansion as Eq. (19) can be expressed as

$$
\begin{aligned}
\frac{\partial^{l} G_{3 D}(\mathbf{x} ; \mathbf{y})}{\partial y_{1}^{l}} & =\frac{-1}{4 \pi} \sum_{k_{1}=0}^{l} C_{l}^{k_{1}}\left(\frac{\partial \varphi(\mathbf{x} ; \mathbf{y})}{\partial y_{1}}\right)^{k_{1}} \exp ^{\varphi(\mathbf{x} ; \mathbf{y})} \frac{\partial^{l-k_{1}}\left(R^{-1}\right)}{\partial y_{1}^{l-k_{1}}} \\
& \approx \frac{-1}{4 \pi} \sum_{k_{1}=0}^{l} C_{l}^{k_{1}}\left(\frac{\partial \varphi(\mathbf{x} ; \mathbf{y})}{\partial y_{1}}\right)^{k_{1}} \exp ^{\varphi(\mathbf{x} ; \mathbf{y})} \frac{\partial^{l-k_{1}}\left(R^{-1}\right)}{\partial y_{1}^{l-k_{1}}}
\end{aligned}
$$

Similar to the derivation of Eq. (14), the $k_{1}$ th-order derivative of $R^{-1}$ can be approximated as follows

$$
\frac{\partial^{k_{1}}\left(R^{-1}\right)}{\partial y_{1}^{k_{1}}} \approx O\left(\frac{1}{R^{k_{1}-1}}\right) \frac{\partial R^{-1}}{\partial y_{1}}
$$

Further, the approximated derivatives of $R^{-1}$ terms can be employed to simplify the right-hand-side of Eq. (20). Thus, we get the far-field approximations to the $(l+2)$ th-order derivative of the Green's function for the three-dimensional flows with $q=1$ as follows,

$$
\frac{\partial^{l}}{\partial y_{1}^{l}}\left(\frac{\partial^{2} G_{3 D}(\mathbf{x} ; \mathbf{y})}{\partial y_{i} \partial y_{j}}\right) \approx\left(\frac{\partial \varphi_{3 D}(\mathbf{x} ; \mathbf{y})}{\partial y_{1}}\right)^{l} \frac{\partial^{2} G_{3 D}(\mathbf{x} ; \mathbf{y})}{\partial y_{i} \partial y_{j}}
$$


The full derivations of the approximations given by Eq. (3) can be obtained by setting the subscript $q=2$ for two-dimensional flows and $q=2,3$ for three-dimensional flows.

\section{Results and Discussion}

\subsection{Two-dimensional convecting vortex}

We use the benchmark flow of two-dimensional convecting vortex to verify the approximations to the derivatives of the Green's function in this subsection. The time-varied velocity and pressure of the convecting vortex are shown as follows

$$
\begin{aligned}
p & =\frac{1}{\gamma}\left(1-a_{2} \exp \left(1-r^{2}\right)\right)^{\frac{\gamma}{\gamma-1}}, \\
u & =U_{1}-a_{0} a_{1} y_{2} \exp \left(\left(1-r^{2}\right) / 2\right), \\
v & =a_{0} a_{1}\left(y_{1}-M t\right) \exp \left(\left(1-r^{2}\right) / 2\right), \\
\rho & =\left(\frac{p}{p_{o}}\right)^{1 / \gamma},
\end{aligned}
$$

where $p_{o}$ is the pressure of the ambient medium at the far-field. $\gamma=1.4$ is the specific heat ratio of air. The quantities are normalized by using the ambient speed of sound $c_{o}$, reference length $\mathrm{L}$ and the density $\rho$ of fluid. We arrange the vortex convecting along the $o-y_{1}$ axis with a velocity of $U_{1} . M a=U_{1} / c_{o}$ is the convecting Mach number. The parameters are given by $a_{0}=1$ and $a_{1}=1 /(2 \pi)$, repsectively. The parameters $a_{2}$ and $r$ are computed by

$$
\begin{aligned}
& a_{2}=(\gamma-1) a_{0}^{2} a_{1}^{2} / 2, \\
& r^{2}=\left(y_{1}-M a t\right)^{2}+y_{2}^{2} .
\end{aligned}
$$

Figure 1 gives the schematic of the pressure field when the two-dimensional convecting vortex is crossing the permeable FW-H integral surface. Here, we take the permeable FW-H integral surface as a square with an edge of 10 unit length, as shown by the dashed edges in Fig. 1. The contours in Fig. 1 show the distribution of the fluctuating pressure $p-p_{o}$, which is normalized by $p_{o}$.

The pressure fluctuation approaches zero monotonically away from the vortex center, as shown in Fig. 2, where the convecting Mach number of the vortex is $M a=0.2$. Thus, the sound pressure should approach to zero at the far-field [15]. However, the computation of the far-field sound pressure using the $\mathrm{FW}-\mathrm{H}$ integral without the surface correction results in significant spurious sound when the vortex is moving across the permeable integral surface, as shown in Fig. 3 where the solid line shows the acoustic presssure computed by using the FW-H surface integral at the position $\mathbf{x}=(100,0)$, which is totally spurious sound.

The surface correction integral given by Eq. (2) and its approximation at the far-field given by Eq. (10) are shown by dashed line and dash-dotted line in Fig. 3 , respectively. For convenience of comparison, we plot the minus of the surface correction integral, i.e. $-I_{Q}$, in Fig. 3. It is clear that the surface correction integrals computed with both the original derivatives of the Green's function (Eq. (2)) and the approximated derivatives of the Green's function (Eq. (10)) can accurately 
estimate the spurious sound generated by the vortex crossing the integral surface. The results shown by Fig. 3 indicate that the approximations of Eq. (3) are correct. To further verify the approximations to the derivatives of the Green's function, we compare the first three terms in Eqs. (2) and (10) in details as follows.

We note the first, second and third terms in Eq. (2) by

$$
\begin{aligned}
& p_{1}^{\prime}(\mathbf{x} ; \omega)=-\int_{f=0}\left(\frac{-i U_{1}}{\omega}\right) T_{i j}(\mathbf{y}, \omega)\left(\frac{\partial^{2} G(\mathbf{x} ; \mathbf{y})}{\partial y_{i} \partial y_{j}}\right) \mathrm{d} S \\
& p_{2}^{\prime}(\mathbf{x} ; \omega)=-\int_{f=0}\left(\frac{-i U_{1}}{\omega}\right)^{2} T_{i j}(\mathbf{y}, \omega) \frac{\partial}{\partial y_{1}}\left(\frac{\partial^{2} G(\mathbf{x} ; \mathbf{y})}{\partial y_{i} \partial y_{j}}\right) \mathrm{d} S \\
& p_{3}^{\prime}(\mathbf{x} ; \omega)=-\int_{f=0}\left(\frac{-i U_{1}}{\omega}\right)^{3} T_{i j}(\mathbf{y}, \omega) \frac{\partial^{2}}{\partial y_{1}^{2}}\left(\frac{\partial^{2} G(\mathbf{x} ; \mathbf{y})}{\partial y_{i} \partial y_{j}}\right) \mathrm{d} S
\end{aligned}
$$

and the first, second and third terms in Eq. (10) by

$$
\begin{aligned}
& p_{1, \text { appr }}^{\prime}(\mathbf{x} ; \omega)=-\int_{f=0}\left(\frac{-i U_{1}}{\omega}\right) T_{i j}(\mathbf{y}, \omega)\left(\frac{\partial^{2} G(\mathbf{x} ; \mathbf{y})}{\partial y_{i} \partial y_{j}}\right) \mathrm{d} S \\
& p_{2, \text { appr }}^{\prime}(\mathbf{x} ; \omega)=-\int_{f=0}\left(\frac{-i U_{1}}{\omega}\right)^{2} T_{i j}(\mathbf{y}, \omega) \frac{\partial \varphi}{\partial y_{1}}\left(\frac{\partial^{2} G(\mathbf{x} ; \mathbf{y})}{\partial y_{i} \partial y_{j}}\right) \mathrm{d} S, \\
& p_{3, \text { appr }}^{\prime}(\mathbf{x} ; \omega)=-\int_{f=0}\left(\frac{-i U_{1}}{\omega}\right)^{3} T_{i j}(\mathbf{y}, \omega)\left(\frac{\partial \varphi}{\partial y_{1}}\right)^{2}\left(\frac{\partial^{2} G(\mathbf{x} ; \mathbf{y})}{\partial y_{i} \partial y_{j}}\right) \mathrm{d} S,
\end{aligned}
$$

where we use the subscript "appr" to indicate the approximated computation of the derivatives of the Green's function in Eq. (10). The first terms in Eq. (2) and Eq. (10) are exactly the same since the approximation to the derivative of the Green's function is not used. The differences between the second and third terms in Eq. (2) and Eq. (10) are that the approximations to the derivatives of the Green's function are used in Eq. (10), as shown in Eqs. (26), (29) and (27), (30), respectively. We verify the approximations to the derivatives of the Green's function by comparing the second terms (Eqs. (26), (29)) and the third terms (Eqs. (27), (30)) at different observer distances, different directions, and different convecting Mach numbers.

Figure 4 shows the sound pressures corresponding to the second and third terms at different observer distances $S=\left|\mathbf{x}-\mathbf{Y}_{\mathbf{0}}\right|$, where $\mathbf{x}$ and $\mathbf{Y}_{\mathbf{0}}$ are the observer position and the initial central position of the convecting vortex, respectively. We set the initial central position of the convecting vortex at $\mathbf{Y}_{\mathbf{0}}=\mathbf{0}$ in this work. The central position of the convecting vortex $\mathbf{Y}$ moves along the $o-y_{1}$ axis. The sound pressures at the non-time dimensional time $t=120$ are plotted in Fig. 4 . At this time moment of $t=120$, the central position of the convecting vortex is at $\mathbf{Y}=(24.0,0.0)$. For the case reported in Fig. 4, the convecting Mach number of the vortex is $M a=0.2$ and the observers are distributed along the line with $\theta=0^{\circ}$, where $\theta$ is defined as the angle between the observer position and the $o-y_{1}$ axis, as shown in Fig. 1. The variations of the sound pressure computed by using the Eqs. (26) and (29) are shown in Fig. 4(a). The results indicate that the sound pressure 
$p_{2 \text {,appr }}^{\prime}$ approaches to the sound pressure $p_{2}^{\prime}$ as the observer distance $S$ increases. The difference between the sound pressure $p_{2, \text { appr }}^{\prime}$ and $p_{2}^{\prime}$ is less than $5 \%$ of $I_{Q}(\mathbf{x})$ at the same position when $S>53$, which indicates that the right-hand side of Eq. (3) gives reasonable approximation to its left-hand-side when $l=1$ when the observer distance $S>53$. The variations of the sound pressure computed by using the Eqs. (27) and (30) are shown in Fig. 4(b). Similar to that corresponding to the second term, the sound pressure $p_{3 \text {,appr }}^{\prime}$ approaches to the sound pressure $p_{3}^{\prime}$ as the observer distance $S$ increases. The difference between the sound pressure $p_{3 \text {,appr }}^{\prime}$ and $p_{3}^{\prime}$ is less than $5 \%$ of $I_{Q}(\mathbf{x})$ at the same position when $S>35$, which indicates that the right-hand side of Eq. (3) gives reasonable approximation to its left-handside when $l=2$ when the observer distance $S>35$. The results also indicate that the approximation on the left-hand-side of Eq. (3) converges to its right-hand-side faster with $l=2$ than that with $l=1$.

Figures 5 and 6 show the variations of the sound pressures with the observer distances at the convecting Mach numbers of $M a=0.3$ and $M a=0.4$, respectively. The observers are distributed along the line with $\theta=0^{\circ}$. We plot the sound pressures at the same non-time dimensional time $t=120$ with that as the case in Fig. 4 . At this time instant of $t=120$, the central position of the convecting vortex is at $\mathbf{Y}=(36.0,0.0)$ for the case $M a=0.3$ and $\mathbf{Y}=(48.0,0.0)$ for the case $M a=0.4$. For the case $M a=0.3$, the variations of the sound pressure computed by using the Eqs. (26) and (29) are shown in Fig. 5(a). Similar to Fig. 4(a), the sound pressure $p_{2, \text { appr }}^{\prime}$ approaches to the sound pressure $p_{2}^{\prime}$ as the observer distance $S$ increases. The difference between the sound pressure $p_{2, \text { appr }}^{\prime}$ and $p_{2}^{\prime}$ is less than $5 \%$ of $I_{Q}(\mathbf{x})$ at the same position when $S>71$. The variations of the sound pressure computed by using the Eqs. (27) and (30) are shown in Fig. 5(b). The sound pressure $p_{3, \text { appr }}^{\prime}$ approaches to the sound pressure $p_{3}^{\prime}$ as the observer distance $S$ increases. The difference between the sound pressure $p_{3, \text { appr }}^{\prime}$ and $p_{3}^{\prime}$ is less than $5 \%$ of $I_{Q}(\mathbf{x})$ at the same position when $S>56$. The results also support the conclusion that the approximation on the lefthand-side of Eq. (3) approaches to its right-hand-side more rapidly with $l=2$ than that with $l=1$. Comparing with these plotted in Fig. 4, the results indicate that the approximations on the left-hand-side of Eq. (3) approach to its right-hand-side more slowly with $M a=0.3$ than that with $M a=0.2$. For the case with $M a=0.4$, the variations of the sound pressure computed by using the Eqs. (26) and (29) are shown in Fig. 6(a). Similar to these plotted in Figs. 4(a) and 5(a), the sound pressure $p_{2, \text { appr }}^{\prime}$ approaches to the sound pressure $p_{2}^{\prime}$ as the observer distance $S$ increases. The variations of the sound pressure computed by using the Eqs. (27) and (30) are shown in Figure $6(\mathrm{~b})$. The sound pressure $p_{3, \text { appr }}^{\prime}$ approaches to the sound pressure $p_{3}^{\prime}$ as the observer distance $S$ increases. All these results support the conclusion that the approximation on the left-hand-side of Eq. (3) converges to its right-handside faster with $l=2$ than that with $l=1$. Comparing with these plots in Fig. 4 and Fig. 5, the results also indicate that the convergence of approximations on the left-hand-side of Eq. (3) to its right-hand-side becomes slow as the convecting Mach number increases.

Figures 7 and 8 show the variations of the sound pressures with the observer distances along the directions with $\theta=45^{\circ}$ and $\theta=90^{\circ}$, respectively. The convecting Mach number is set as $M a=0.2$. We plot the sound pressures at the same non-time 
dimensional time $t=120$ with that for the case shown in Figure 4 . For the case with $\theta=45^{\circ}$, the variations of the sound pressure computed by using the Eqs. (26) and (29) are shown in Fig. 7(a). Similar to these plotted in Fig. 4(a), the sound pressure $p_{2, \text { appr }}^{\prime}$ converges to the sound pressure $p_{2}^{\prime}$ as the observer distance $S$ increases. The difference between the sound pressure $p_{2 \text {,appr }}^{\prime}$ and $p_{2}^{\prime}$ is less than $5 \%$ of $I_{Q}(\mathbf{x})$ at the same position when $S>42$. The variations of the sound pressure computed by using the Eqs. (27) and (30) are shown in Figure 7(b). The sound pressure $p_{3 \text {,appr }}^{\prime}$ converges to the sound pressure $p_{3}^{\prime}$ as the observer distance $S$ increases. The difference between the sound pressure $p_{3 \text {,appr }}^{\prime}$ and $p_{3}^{\prime}$ is less than $5 \%$ of $I_{Q}(\mathbf{x})$ at the same position when $S>39$. For the case with $\theta=90^{\circ}$, the variations of the sound pressure computed by using the Eqs. (26) and (29) are shown in Figure 8(a). The sound pressure $p_{2, \text { appr }}^{\prime}$ converges to the sound pressure $p_{2}^{\prime}$ as the observer distance $S$ increases. The difference between the sound pressure $p_{2 \text {,appr }}^{\prime}$ and $p_{2}^{\prime}$ is less than $5 \%$ of $I_{Q}(\mathbf{x})$ at the same position when $S>42$. The variations of the sound pressure computed by using the Eqs. (27) and (30) are shown in Figure 8(b). The sound pressure $p_{3, \text { appr }}^{\prime}$ converges to the sound pressure $p_{3}^{\prime}$ as the observer distance $S$ increases. The difference between the sound pressure $p_{3, \text { appr }}^{\prime}$ and $p_{3}^{\prime}$ is less than $5 \%$ of $I_{Q}(\mathbf{x})$ at the same position when $S>37$. All these results support the conclusion that the approximation on the left-hand-side of Eq. (3) converges to its right-hand-side faster with $l=2$ than that with $l=1$. Compare with these plotted in Figures 4, 7, and 8 , the results indicate that the approximations on the left-hand-side of Eq. (3) converge to its right-hand-side become faster as the directive angle $\theta$ increases.

\subsection{Co-rotating vortex pair}

In this subsection we use the benchmark flow of a co-rotating vortex pair to verify the approximations to the derivatives of the Green's function. The co-rotating vortex pair is a classic model for studying the jet engine noise generated from the interaction of coherent structures [20]. A co-rotating vortex pair can be modeled by two line vortices of equal strength separated by the distance $2 d$ as is shown in Fig. 9 [21]. The sound generated by the co-rotating vortex pair can be computed by modifying the Lighthill's acoustic analogy equation into the vortex sound equation as follows,

$$
\frac{1}{c_{\infty}^{2}} \frac{\partial^{2} p^{\prime}}{\partial t^{2}}-\frac{\partial^{2} p^{\prime}}{\partial x_{i}^{2}}=\rho_{0} \frac{\partial(\omega \times \mathbf{v})_{i}}{\partial x_{i}}
$$

where the sound source other than the divergence of the Lamb vector $\omega \times \mathbf{v}$ are neglected according to the dimensional analysis [22]. The corresponding frequencydomain formulation can be expressed as

$$
k^{2} p^{\prime}+\frac{\partial^{2} p^{\prime}}{\partial x_{i}^{2}}=-\rho_{0} \mathcal{F}\left(\frac{\partial(\omega \times \mathbf{v})_{i}}{\partial x_{i}}\right)
$$

where the operator $\mathcal{F}$ defines the Fourier transform. It is noted that although we use the Lighthill's equation here, the corresponding Green's funtion is consistent with the Green's function of the FW-H equation without freestream flow. By using 
the Gauss's theorem, the far-field acoustic pressure can be computed by

$$
\frac{p^{\prime}}{\rho_{0}}=\int_{V} \mathcal{F}\left((\omega \times \mathbf{v})_{i}\right) \frac{\partial G_{2 D}(\mathbf{x} ; \mathbf{y})}{\partial y_{i}} \mathrm{~d} V
$$

For a co-rotating vortex pair, the Lamb vector $\omega \times \mathbf{v}$ can be expressed as

$\omega \times \mathbf{v}=-\Omega \Gamma \mathbf{y}^{(\nu)}\left(\delta\left(y_{1}-y_{1}^{(\nu)}\right) \delta\left(y_{2}-y_{2}^{(\nu)}\right)-\delta\left(y_{1}+y_{1}^{(\nu)}\right) \delta\left(y_{2}+y_{2}^{(\nu)}\right)\right)$

where $\Omega=\frac{\Gamma}{4 \pi d^{2}}$ is the angular velocity of the vortex pair and $\pm \mathbf{y}^{(\nu)}=$ $\pm(d \cos (\Omega t), d \sin (\Omega t))$ represent the location of the vortex pair. By using the sifting property of the Dirac delta function, the frequency-domain far-field acoustic pressure generated by the vortex pair can be obtained by the acoustic pressure formulation as follows,

$$
\begin{aligned}
\frac{p^{\prime}}{\rho_{0}}=- & \mathcal{F}\left(\Omega \Gamma d \cos (\Omega t)\left(\left.\frac{\partial G_{2 D}}{\partial y_{1}}\right|_{\mathbf{y}=\mathbf{y}^{(\nu)}}-\left.\frac{\partial G_{2 D}}{\partial y_{1}}\right|_{\mathbf{y}=-\mathbf{y}^{(\nu)}}\right)\right)- \\
& \mathcal{F}\left(\Omega \Gamma d \sin (\Omega t)\left(\left.\frac{\partial G_{2 D}}{\partial y_{2}}\right|_{\mathbf{y}=\mathbf{y}^{(\nu)}}-\left.\frac{\partial G_{2 D}}{\partial y_{2}}\right|_{\mathbf{y}=-\mathbf{y}^{(\nu)}}\right)\right) .
\end{aligned}
$$

To verify the approximations to the derivatives of the Green's function, we first conduct Taylor's expansion for the Green's function,

$$
G_{2 D}=G_{0}+\sum_{j} \frac{1}{j !}\left(y_{m} \frac{\partial}{\partial y_{m}}\right)^{j} G_{2 D}(\mathbf{x} ; \mathbf{y})
$$

By using the approximations to the derivatives of the Green's function given by Eq. (3), we can approximate the expansion of the Green's function as follows

$$
G_{2 D}=G_{0}+\sum_{j} \frac{1}{j !}\left(y_{m} \frac{\partial \varphi_{0}}{\partial y_{m}}\right)^{j} G_{0}
$$

where $G_{0}$ and $\frac{\partial \varphi_{0}}{\partial y_{m}}$ are the Green's function $G$ and space derivatives of the phase function $\frac{\partial \varphi}{\partial y_{m}}$ at the origin. By replacing the Green's function in Eq. (35) with the approximations in Eq. (37), we can obtain the approximated acoustic pressure formulation as follows

$$
\frac{p^{\prime}}{\rho_{0}} \approx \sum_{j=1}^{n} \frac{-\Omega \Gamma G_{0}}{(j-1) !} \mathcal{F}\left(\left(y_{1}^{(\nu)} \frac{\partial \varphi_{0}}{\partial y_{1}}+y_{2}^{(\nu)} \frac{\partial \varphi_{0}}{\partial y_{2}}\right)^{j}\right)\left(1+(-1)^{j}\right) .
$$

It is noted that the first nonzero term in Eq. (38) is the quadrupole term, which confirms that the quadrupole source term dominates the acoustic pressure generated by the co-rotating vortex pair [23].

Figure 10 compares the far-field acoustic pressure computed by using the approximated acoustic pressure formulation Eq. (38) with that computed by the acoustic 
pressure formulation Eq. (35). For the results reported in Fig. 10, the observer is located at $\left(\frac{100 \Omega}{2 \pi}, \frac{100 \Omega}{2 \pi}\right)$ which is sufficient far away from the center of the co-rotating vortex pair. The density of fluid is taken as $1 \mathrm{~kg} / \mathrm{m}^{3}$. The sound speed is set as $c=100 \mathrm{~m} / \mathrm{s}$, which is sufficient large compared to the characteristic velocity of the vortex $\mathrm{U}=\Omega d$. The distance between the two vorticies is $2 d=0.2 m$ and the angular velocity is $\Omega=2 \pi \mathrm{rad} / \mathrm{s}$. The black solid line in Fig. 10 is the result computed by using the acoustic pressure formulation Eq. (35) while the red circle is that computed by using the approximated acoustic pressure formualtion (Eq. (38) with $\mathrm{n}=2$ ). It is observed that the approximated acoustic pressure formulation (Eq. (38)) gives a good approximation to the result of vortex acoustic pressure (Eq. (35))), which indicates that the approximations to the derivatives of the Green's function given by Eq. (3) is correct.

\subsection{Approximations to the integrals of the Green's function}

The approximations to the integrals of the Green's function have been used by Zhou et al. [15] to fixed the divergence problem of the frequency-domain surface correction integral [17]. However, the detailed derivations of the approximations to the integrals of the Green's function have not been provided. In this subsection we show that the approximations to the integrals of the Green's function can be derived based on the approximations to the derivatives of the Green's function.

The approximations to the integrals of the Green's function with respect to the variable $y_{1}$ at the far field used in the work of Zhou et al. [15] are given as follows,

$$
\frac{\partial^{2} G^{q, n}(\mathbf{x} ; \mathbf{y})}{\partial y_{i} \partial y_{j}} \approx\left(\frac{\partial \varphi(\mathbf{x} ; \mathbf{y})}{\partial y_{q}}\right)^{-n} \frac{\partial^{2} G(\mathbf{x} ; \mathbf{y})}{\partial y_{i} \partial y_{j}}
$$

where

$$
\begin{aligned}
\frac{\partial^{2} G^{q, n}(\mathbf{x} ; \mathbf{y})}{\partial y_{i} \partial y_{j}} & =I^{q, n}\left(\frac{\partial^{2} G(\mathbf{x} ; \mathbf{y})}{\partial y_{i} \partial y_{j}}\right) \\
& =\int_{\infty}^{y_{q}}\left(\int_{\infty}^{\xi_{n}}\left(\int_{\infty}^{\xi_{n-1}}\left(\cdots \int_{\infty}^{\xi_{3}}\left(\int_{\infty}^{\xi_{2}} \frac{\partial^{2} G\left(\mathbf{x} ; \xi_{1}, y_{2}\right)}{\partial y_{i} \partial y_{j}} d \xi_{1}\right) d \xi_{2} \cdots\right) d \xi_{n-2}\right) d \xi_{n-1}\right) d \xi_{n}
\end{aligned}
$$

is the multiple integral of $\frac{\partial^{2} G(\mathbf{x} ; \mathbf{y})}{\partial y_{i} \partial y_{j}}$ with repect to the variable $y_{q}$. For the $i$ th integral $(i<n)$ from the inner of Eq. (40), the independent variable is $\xi_{i}$ and the upper limit of the integral is $\xi_{i+1}$. When $i=n$, the independent variable is $\xi_{n}$ and the upper limit of the integral is $y_{q}$.

Eq. (39) is obtained through proving the following equation.

$$
\frac{\partial^{2} G^{q, l}(\mathbf{x} ; \mathbf{y})}{\partial y_{i} \partial y_{j}}=\frac{\partial^{2}\left(\left(\frac{\partial \varphi(\mathbf{x} ; \mathbf{y})}{\partial y_{q}}\right)^{-l} G(\mathbf{x} ; \mathbf{y})\right)}{\partial y_{i} \partial y_{j}}
$$

We give the details of the derivation of Eq. (41) for the two-dimensional flows by using the mathematical induction method and the approximations as Eq. (3). 
The derivation of the Eq. (41) for the three-dimensional flows can be obtained in a similar way.

To use the mathematical induction method, we first prove that Eq. (41) is valid when $l=1$ and $q=1$. For $l=1$ and $q=1$, Eq. (40) reduces to

$$
\frac{\partial^{2} G_{2 D}^{1,1}(\mathbf{x} ; \mathbf{y})}{\partial y_{i} \partial y_{j}}=\int_{\infty}^{y_{1}}\left(\left.\frac{\partial^{2} G_{2 D}(\mathbf{x} ; \mathbf{y})}{\partial y_{i} \partial y_{j}}\right|_{y_{1}=\xi_{1}}\right) d \xi_{1}
$$

After transforming the partial derivative with respect to $\mathbf{y}$ to $\mathbf{x}$ and using integration by parts, the right-hand-side of Eq. (42) becomes

$$
\begin{aligned}
\frac{\partial^{2} G_{2 D}^{1,1}(\mathbf{x} ; \mathbf{y})}{\partial y_{i} \partial y_{j}}= & \frac{\partial^{2}}{\partial x_{i} \partial x_{j}} \int_{\infty}^{y_{1}} \frac{i}{4 \beta}\left(\frac{2 \beta^{2}}{\pi k R}\right)^{1 / 2} \exp ^{\varphi\left(\mathbf{x} ; \xi_{1}, y_{2}\right)} d \xi_{1} \\
= & \frac{\partial^{2}}{\partial x_{i} \partial x_{j}} \int_{\infty}^{y_{1}} \frac{\partial}{\partial \xi_{1}}\left[\frac{i}{4 \beta}\left(\frac{2 \beta^{2}}{\pi k R}\right)^{1 / 2}\left(\frac{\partial \varphi\left(\mathbf{x} ; \xi_{1}, y_{2}\right)}{\partial \xi_{1}}\right)^{-1} \exp ^{\varphi\left(\mathbf{x} ; \xi_{1}, y_{2}\right)}\right] d \xi_{1}- \\
& \frac{\partial^{2}}{\partial x_{i} \partial x_{j}} \int_{\infty}^{y_{1}}\left[\left(\frac{\partial \varphi\left(\mathbf{x} ; \xi_{1}, y_{2}\right)}{\partial \xi_{1}}\right)^{-1} \exp ^{\varphi\left(\mathbf{x} ; \xi_{1}, y_{2}\right)}\right] \frac{\partial}{\partial \xi_{1}}\left(\frac{i}{4 \beta}\left(\frac{2 \beta^{2}}{\pi k R}\right)^{1 / 2}\right) d \xi_{1}
\end{aligned}
$$

From Eq. (15), we know that the last term on the right-hand-side of Eq. (43) is ignorable compared with the left-hand-side of Eq. (43), thus we have

$$
\frac{\partial^{2} G_{2 D}^{1,1}(\mathbf{x} ; \mathbf{y})}{\partial y_{i} \partial y_{j}} \approx \frac{\partial^{2}}{\partial x_{i} \partial x_{j}} \int_{\infty}^{y_{1}} \frac{\partial}{\partial \xi_{1}}\left[\frac{i}{4 \beta}\left(\frac{2 \beta^{2}}{\pi k R}\right)^{1 / 2}\left(\frac{\partial \varphi\left(\mathbf{x} ; \xi_{1}, y_{2}\right)}{\partial \xi_{1}}\right)^{-1} \exp ^{\varphi\left(\mathbf{x} ; \xi_{1}, y_{2}\right)}\right] d \xi_{1}
$$

Considering the limitation of the Green's function at infinity and transforming the partial derivative with respect to $\mathbf{x}$ back to $\mathbf{y}$, Eq. (44) becomes

$$
\begin{aligned}
\frac{\partial^{2} G_{2 D}^{1,1}(\mathbf{x} ; \mathbf{y})}{\partial y_{i} \partial y_{j}} & \approx \frac{\partial^{2}}{\partial y_{i} \partial y_{j}}\left[\frac{i}{4 \beta}\left(\frac{2 \beta^{2}}{\pi k R}\right)^{1 / 2}\left(\frac{\partial \varphi(\mathbf{x} ; \mathbf{y})}{\partial y_{1}}\right)^{-1} \exp ^{\varphi(\mathbf{x} ; \mathbf{y})}\right] \\
& =\frac{\partial^{2}\left(\left(\frac{\partial \varphi(\mathbf{x} ; \mathbf{y})}{\partial y_{1}}\right)^{-1} G_{2 D}(\mathbf{x} ; \mathbf{y})\right)}{\partial y_{i} \partial y_{j}},
\end{aligned}
$$

which proves that Eq. (41) is valid when $l=1$ and $q=1$.

According to the procedures of the mathematical induction method, we assume that Eq. (41) is valid when $l=h$ and $q=1$

$$
\frac{\partial^{2} G_{2 D}^{1, h}(\mathbf{x} ; \mathbf{y})}{\partial y_{i} \partial y_{j}} \approx \frac{\partial^{2}\left[\left(\frac{\partial \varphi(\mathbf{x} ; \mathbf{y})}{\partial y_{1}}\right)^{-h} G_{2 D}(\mathbf{x} ; \mathbf{y})\right]}{\partial y_{i} \partial y_{j}}
$$


According to Eqs. (40) and (46), we have

$$
\frac{\partial^{2} G_{2 D}^{1, h+1}(\mathbf{x} ; \mathbf{y})}{\partial y_{i} \partial y_{j}} \approx \int_{\infty}^{y_{h}}\left(\left.\frac{\partial^{2}\left[\left(\frac{\partial \varphi(\mathbf{x} ; \mathbf{y})}{\partial y_{1}}\right)^{-l} G_{2 D}(\mathbf{x} ; \mathbf{y})\right]}{\partial y_{i} \partial y_{j}}\right|_{y_{1}=\xi_{1}}\right) d \xi_{1}
$$

Using integration by parts, we reform Eq. (47) as follows

$$
\begin{aligned}
& \frac{\partial^{2} G_{2 D}^{1, h+1}(\mathbf{x} ; \mathbf{y})}{\partial y_{i} \partial y_{j}} \approx \frac{\partial^{2}}{\partial x_{i} \partial x_{j}} \int_{\infty}^{y_{1}}\left(\left.\frac{i}{4 \beta}\left(\frac{\partial \varphi(\mathbf{x} ; \mathbf{y})}{\partial y_{1}}\right)^{-h}\left(\frac{2 \beta^{2}}{\pi k R}\right)^{1 / 2} \exp ^{\varphi(\mathbf{x} ; \mathbf{y})}\right|_{y_{1}=\xi_{1}}\right) d \xi_{1} \\
& =\frac{\partial^{2}}{\partial x_{i} \partial x_{j}} \int_{\infty}^{y_{1}}\left\{\left.\frac{\partial}{\partial y_{1}}\left[\frac{i}{4 \beta}\left(\frac{\partial \varphi(\mathbf{x} ; \mathbf{y})}{\partial y_{1}}\right)^{-h}\left(\frac{2 \beta^{2}}{\pi k R}\right)^{1 / 2}\left(\frac{\partial \varphi(\mathbf{x} ; \mathbf{y})}{\partial y_{1}}\right)^{-1} \exp ^{\varphi(\mathbf{x} ; \mathbf{y})}\right]\right|_{y_{1}=\xi_{1}}\right\} d \xi_{1}- \\
& \frac{\partial^{2}}{\partial x_{i} \partial x_{j}} \int_{\infty}^{y_{1}}\left\{\left.\left[\left(\frac{\partial \varphi(\mathbf{x} ; \mathbf{y})}{\partial y_{1}}\right)^{-1} \exp ^{\varphi(\mathbf{x} ; \mathbf{y})}\right] \frac{\partial}{\partial y_{1}}\left[\frac{i}{4 \beta}\left(\frac{\partial \varphi(\mathbf{x} ; \mathbf{y})}{\partial y_{1}}\right)^{-h}\left(\frac{2 \beta^{2}}{\pi k R}\right)^{1 / 2}\right]\right|_{y_{1}=\xi_{1}}\right\} d \xi_{1} .
\end{aligned}
$$

From Eq. (15), we know that the last term on the right-hand-side of Eq. (48) is ignorable compared with the left-hand-side of the Eq. (48). Simliar to the derivation of Eq. (45), Eq. (48) could be approximated by

$$
\frac{\partial^{2} G_{2 D}^{1, h+1}(\mathbf{x} ; \mathbf{y})}{\partial y_{i} \partial y_{j}} \approx \frac{\partial^{2}}{\partial y_{i} \partial y_{j}}\left[\frac{i}{4 \beta}\left(\frac{2 \beta^{2}}{\pi k R}\right)^{1 / 2}\left(\frac{\partial \varphi(\mathbf{x} ; \mathbf{y})}{\partial y_{1}}\right)^{-(h+1)} \exp ^{\varphi(\mathbf{x} ; \mathbf{y})}\right]
$$

Eq. (49) shows that Eq. (41) is valid when $l=h+1$ and $q=1$.

By using Leibniz product rule, the right-hand-side of Eq. (41) equals to

$$
\begin{aligned}
& \frac{\partial^{2}}{\partial y_{i} \partial y_{j}}\left[\frac{i}{4 \beta}\left(\frac{2 \beta^{2}}{\pi k}\right)^{1 / 2}\left(R^{-1 / 2}\left(\frac{\partial \varphi(\mathbf{x} ; \mathbf{y})}{\partial y_{1}}\right)^{-l}\right) \exp ^{\varphi(\mathbf{x} ; \mathbf{y})}=\right. \\
& \frac{i}{4 \beta}\left(\frac{2 \beta^{2}}{\pi k}\right)^{1 / 2}\left[R^{-1 / 2}\left(\frac{\partial \varphi(\mathbf{x} ; \mathbf{y})}{\partial y_{1}}\right)^{-l} \exp ^{\varphi(\mathbf{x} ; \mathbf{y})}\left(\left(\frac{\partial \varphi(\mathbf{x} ; \mathbf{y})}{\partial y_{i}}\right)\left(\frac{\partial \varphi(\mathbf{x} ; \mathbf{y})}{\partial y_{j}}\right)+o(1)\right)\right]+ \\
& \frac{i}{4 \beta}\left(\frac{2 \beta^{2}}{\pi k}\right)^{1 / 2}\left[\frac{\partial\left(R^{-1 / 2}\left(\frac{\partial \varphi(\mathbf{x} ; \mathbf{y})}{\partial y_{1}}\right)^{-l}\right)}{\partial y_{i}} \frac{\partial\left(\exp ^{\varphi(\mathbf{x} ; \mathbf{y})}\right)}{\partial y_{j}}\right]+ \\
& \frac{i}{4 \beta}\left(\frac{2 \beta^{2}}{\pi k}\right)^{1 / 2}\left[\frac{\partial\left(R^{-1 / 2}\left(\frac{\partial \varphi(\mathbf{x} ; \mathbf{y})}{\partial y_{1}}\right)^{-l}\right)}{\partial y_{j}} \frac{\partial\left(\exp ^{\varphi(\mathbf{x} ; \mathbf{y})}\right)}{\partial y_{i}}\right]+ \\
& \frac{i}{4 \beta}\left(\frac{2 \beta^{2}}{\pi k}\right)^{1 / 2}\left[\frac{\partial^{2}\left(R^{-1 / 2}\left(\frac{\partial \varphi(\mathbf{x} ; \mathbf{y})}{\partial y_{1}}\right)^{-l}\right)}{\partial y_{i} \partial y_{j}} \exp ^{\varphi(\mathbf{x} ; \mathbf{y})} \cdot\right.
\end{aligned}
$$


In the far field, $\frac{\partial \varphi(\mathbf{x} ; \mathbf{y})}{\partial y_{i}}$ is of order $O(1)$ and the derivative of $R^{-1 / 2}\left(\frac{\partial \varphi(\mathbf{x} ; \mathbf{y})}{\partial y_{1}}\right)^{-l}$ can be expressed by $o\left(R^{-1 / 2}\left(\frac{\partial \varphi(\mathbf{x} ; \mathbf{y})}{\partial y_{1}}\right)^{-l}\right)$. Thus, the last three terms of Eq. (50) are ignorable compared with the first term on the right-hand-side. We ignore the derivatives corresponding to $\left(\frac{\partial \varphi(\mathbf{x} ; \mathbf{y})}{\partial y_{1}}\right)^{-l}$ and obtain

$$
\begin{array}{r}
\frac{\partial^{2}}{\partial y_{i} \partial y_{j}}\left[\frac{i}{4 \beta}\left(\frac{2 \beta^{2}}{\pi k}\right)^{1 / 2}\left(R^{-1 / 2}\left(\frac{\partial \varphi(\mathbf{x} ; \mathbf{y})}{\partial y_{1}}\right)^{-l}\right) \exp ^{\varphi(\mathbf{x} ; \mathbf{y})}\right] \\
\approx\left(\frac{\partial \varphi(\mathbf{x} ; \mathbf{y})}{\partial y_{1}}\right)^{-l} \frac{\partial^{2}}{\partial y_{i} \partial y_{j}}\left[\frac{i}{4 \beta}\left(\frac{2 \beta^{2}}{\pi k}\right)^{1 / 2} R^{-1 / 2} \exp ^{\varphi(\mathbf{x} ; \mathbf{y})}\right] \\
=\left(\frac{\partial \varphi(\mathbf{x} ; \mathbf{y})}{\partial y_{1}}\right)^{-l} \frac{\partial^{2} G_{2 D}}{\partial y_{i} \partial y_{j}} .
\end{array}
$$

Finally, substituting Eq. (51) in Eq. (49) yields to Eq. (39). Thus, the approximations to the integral of the Green's function are derivated.

We use a homogeneous vortical flow with a uniform Lighthill stress tensor to verify the far-field approximations to the integrals of the Green's function. We define the uniform Lighthill stress tensor as $T_{i j}=A \cos (2 \pi f t)$ where $A$ is $1 / \mathrm{s}^{2}$. The uniform vortical flow moves within a domain $V$ of $[0,10] \times[0,10]$. The frequency $f$ of is taken as $1 \mathrm{~Hz}$. The sound speed is taken as $c_{0}=340 \mathrm{~m} / \mathrm{s}$ and the density is taken as $\rho=1 \mathrm{~kg} / \mathrm{m}^{3}$. The far-field observer is located at $(340000 \mathrm{~m}, 340000 \mathrm{~m})$, which is sufficient large compared to the wavelength of sound. The freestream Mach number is 0.3 along the $o-y_{1}$ axis. The contribution from the Lighthill stress tensor to the far-field sound can be computed by using the quadrupole source term in the FW-H integral as follows

$$
I_{Q, \mathrm{FW}-\mathrm{H}}=\int_{V} T_{i j} \frac{\partial^{2} G_{2 D}(\mathbf{x} ; \mathbf{y})}{\partial y_{i} \partial y_{j}} \mathrm{~d} \mathbf{y}
$$

By using the far-field approximations to the integrals of the Green's function (Eq. (39)), the far-field sound can be approximated by

$$
\begin{aligned}
I_{Q, \text { appr }} & =T_{i j} \int_{V} \frac{\partial^{2} G_{2 D}(\mathbf{x} ; \mathbf{y})}{\partial y_{i} \partial y_{j}} \mathrm{~d} \mathbf{y} \\
& =\frac{1}{2} T_{i j} \int_{V} \frac{\partial}{\partial y_{1}}\left(\left(\frac{\partial \varphi_{2 D}}{\partial y_{1}}\right)^{-1} \frac{\partial^{2} G_{2 D}(\mathbf{x} ; \mathbf{y})}{\partial y_{i} \partial y_{j}}\right)+\frac{\partial}{\partial y_{2}}\left(\left(\frac{\partial \varphi_{2 D}}{\partial y_{2}}\right)^{-1} \frac{\partial^{2} G_{2 D}(\mathbf{x} ; \mathbf{y})}{\partial y_{i} \partial y_{j}}\right) \mathrm{d} \mathbf{y} \\
& =\frac{1}{2} T_{i j} \int_{S}\left(\frac{\partial \varphi_{2 D}}{\partial y_{q}}\right)^{-1} \frac{\partial^{2} G_{2 D}(\mathbf{x} ; \mathbf{y})}{\partial y_{i} \partial y_{j}} n_{q} \mathrm{~d} \mathbf{y} .
\end{aligned}
$$

Figure 11 shows the results calculate by using Eqs. (52) and (53), respectively. The results computed by using the surface integral (Eq. (53)) matches well with the volume integral (Eq. (52)), which indicates that the approximation to the integrals of the Green's function is valid. 


\section{Conclusion}

The computation of the high-order derivatives of the Green's function for the FW$\mathrm{H}$ equation are required in eliminating the spurious sound associated with the quadrupole sources. The approximations to the derivatives of the Green's function in the frequency domain have been used without derivatives in the previous work. This work provides the detailed derivatives of the approximations to the derivatives of the Green's function. The essential expressions and formulations associated with the binomial expression of the derivatives of the Green's function and the far-field condition to obtain the approximations are provided in details. The benchmark flows of two-dimensional convecting vortex and co-rotating vortex pair are used to verify the approximations. The results show that the approximations can be accurate at different Mach numbers and observer directions as long as the distance is large enough. The derivations of the approximations to integrals of the Green's function by using the approximations to the derivertives are also reported in details.

\section{Acknowledgements}

This work is supported by the National Numerical Windtunnel project, and the National Natural Science Foundation of China (Nos. 11922214, 91752118). The computations are conducted on Tianhe-1 at the National Supercomputer Center in Tianjin.

Funding

the National Natural Science Foundation of China (Nos. 11922214, 91752118)

Availability of data and materials

All the data and materials are available in this paper.

Competing interests

The authors declare that they have no competing interests.

Authors' contributions

Zhiteng Zhou provided the derivation and wroten the manuscript. Zhengyu Zang examined the derivation with benchmarks. Shizhao Wang read and revised the manuscript. All prople read and approved the final manuscript.

Author details

${ }^{1}$ Institute of Mechanics, Chinese Academy of Sciences, Beijing, China. ${ }^{2}$ School of Engineering Sciences, University of Chinese Academy of Sciences, Beijing, China.

References

1. Ffowcs Williams, J.E., Hawkings, D.L.: Sound generation by turbulence and surfaces in arbitrary motion. Philosophical Transactions of the Royal Society of London. Series A, Mathematical and Physical Sciences 264(1151), 321-342 (1969)

2. Fosso Pouangué, A., Sanjosé, M., Moreau, S., Daviller, G., Deniau, H.: Subsonic jet noise simulations using both structured and unstructured grids. AIAA Journal 53(1), 55-69 (2015)

3. Shur, M., Spalart, P., Strelets, M.K., Travin, A.: Towards the prediction of noise from jet engines. International Journal of Heat and Fluid Flow 24(4), 551-561 (2003)

4. Poggi, C., Rossetti, M., Bernardini, G., lemma, U., Andolfi, C., Milano, C., Gennaretti, M.: Surrogate models for predicting noise emission and aerodynamic performance of propellers. Aerospace Science and Technology, 107016 (2021)

5. Li, F., Huang, Q., Pan, G., Shi, Y.: Numerical study on hydrodynamic performance and flow noise of a hydrofoil with wavy leading-edge. AIP Advances 11(9), 095105 (2021)

6. Sun, Z., Song, J., An, Y.: Numerical simulation of aerodynamic noise generated by high speed trains. Engineering Applications of Computational Fluid Mechanics 6(2), 173-185 (2012)

7. Mo, H., An, Y., Liu, Q.: Influence of the length of high-speed train on the far-field aeroacoustics characteristics. Chinese Journal of Theoretical and Applied Mechanics 51(5), 1310-1320 (2019)

8. Song, H., Yi, M., Huang, J., Pan, Y., Liu, D.: Numerical method to compute acoustic scattering effect of a moving source. SpringerPlus 5(1), 1-21 (2016)

9. Zhang, Y., Meng, W., Fan, B., Tang, W.: Biomimetic optimization research on wind noise reduction of an asymmetric cross-section bar. SpringerPlus 5(1), 1-12 (2016)

10. Lockard, D.P.: An efficient, two-dimensional implementation of the Ffowcs Williams and Hawkings equation. Journal of Sound and Vibration 229(4), 897-911 (2000)

11. Farassat, F.: Derivation of Formulations 1 and $1 \mathrm{~A}$ of Farassat. Technical report (2007)

12. Zhong, S., Zhang, X.: A sound extrapolation method for aeroacoustics far-field prediction in presence of vortical waves. Journal of Fluid Mechanics 820, 424-450 (2017) 
13. Ikeda, T., Enomoto, S., Yamamoto, K., Amemiya, K.: On the modification of the Ffowcs Williams-Hawkings integration for jet noise prediction. In: 19th AIAA/CEAS Aeroacoustics Conference, p. 2277 (2013)

14. Yao, H.-D., Davidson, L., Eriksson, L.-E.: Noise radiated by low-Reynolds number flows past a hemisphere at $\mathrm{Ma}=$ 0.3. Physics of Fluids 29(7), 076102 (2017)

15. Zhou, Z., Wang, H., Wang, S., He, G.: Lighthill stress flux model for Ffowcs Williams-Hawkings integrals in frequency domain. AIAA Journal, 1-6 (2021)

16. Wang, M., Lele, S.K., Moin, P.: Computation of quadrupole noise using acoustic analogy. AIAA journal 34(11), 2247-2254 (1996)

17. Lockard, D., Casper, J.: Permeable surface corrections for Ffowcs Williams and Hawkings integrals. In: 11th AIAA/CEAS Aeroacoustics Conference, p. 2995 (2005)

18. Nitzkorski, Z., Mahesh, K.: A dynamic end cap technique for sound computation using the Ffowcs Williams and Hawkings equations. Physics of Fluids 26(11), 115101 (2014)

19. Zhou, Z., Wang, H., Wang, S.: Simplified permeable surface correction for frequency-domain Ffowcs Williams and Hawkings integrals. Theoretical and Applied Mechanics Letters, 100259 (2021)

20. Feng, F., Meng, X., Wang, Q.: Sound generation by a pair of co-rotating vortices using spectral acoustic analogy. Journal of Sound and Vibration 469, 115120 (2020)

21. Leweke, T., Le Dizes, S., Williamson, C.H.: Dynamics and instabilities of vortex pairs. Annual Review of Fluid Mechanics 48, 507-541 (2016)

22. Howe, M.S.: Theory of Vortex Sound vol. 33, (2003)

23. Mitchell, B.E., Lele, S.K., Moin, P.: Direct computation of the sound from a compressible co-rotating vortex pair. Journal of Fluid Mechanics 285, 181-202 (1995) 


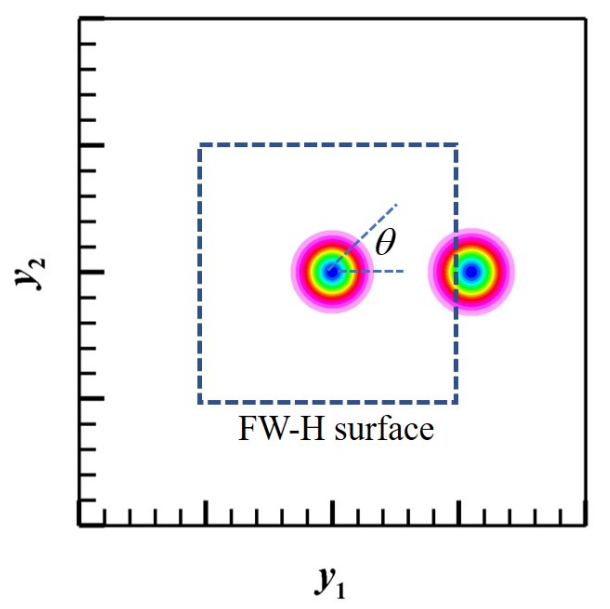

Figure 1 Instantaneous pressure fluctuation field generated by a convecting vortex.

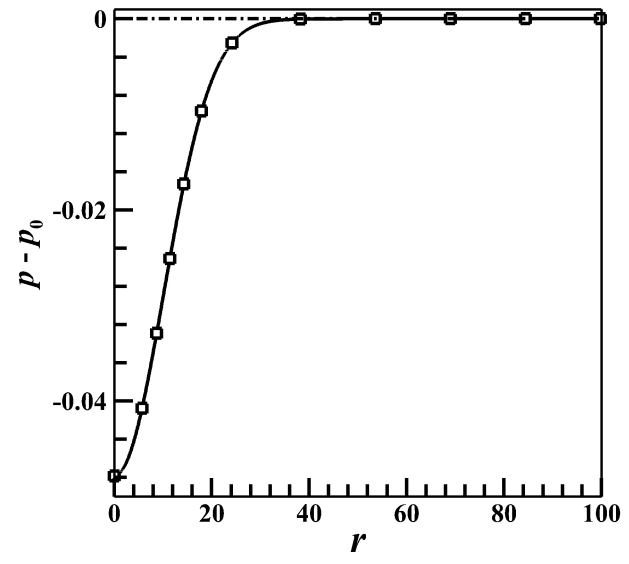

Figure 2 Spaced-varied pressure fluctuation along the streamwise direction ( $y_{1}$-axis) from the vortex center at the freestream Mach number 0.2.

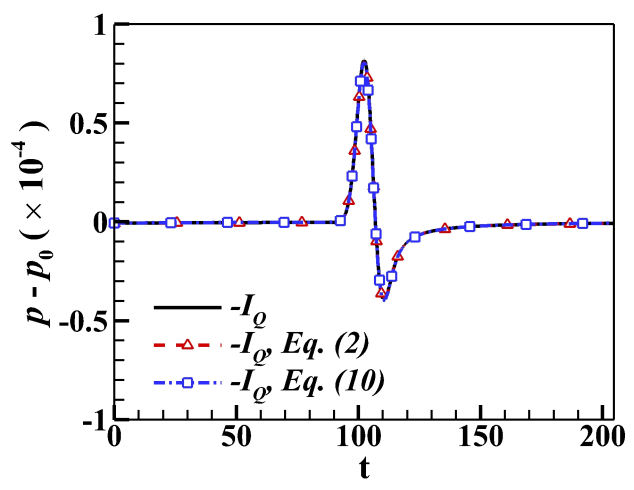

Figure 3 Comparison of the quadrupole correction $\left(I_{Q}\right)$ to the errors produced by the FW-H integral. 
(a)

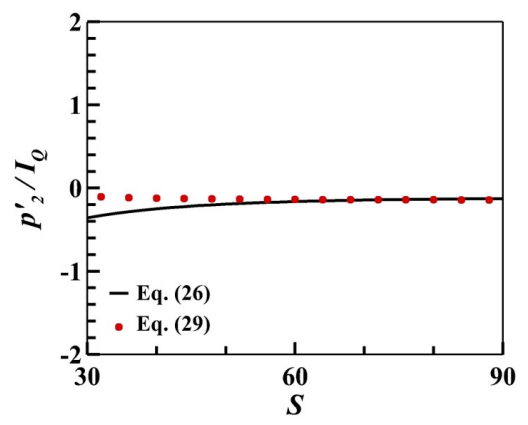

(b)

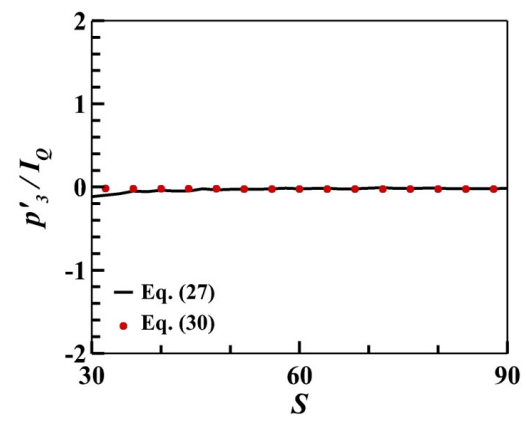

Figure 4 Sound pressures correspond to (a) the second term and (b) the third term at different observer distances $S$. The sound pressures corresponds to the second term are computed by using the Eqs. (26) and (29), respectively. The sound pressures corresponds to the third term are computed by using the Eqs. (27) and (30), respectively. The sound pressures at the non-time dimensional time $t=120$ are plotted. The convecting Mach number of the vortex is $M a=0.2$ and the observers are distributed along the line with $\theta=0^{\circ}$.

(a)

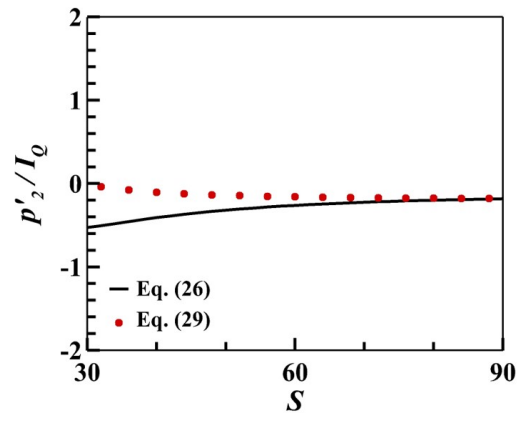

(b)

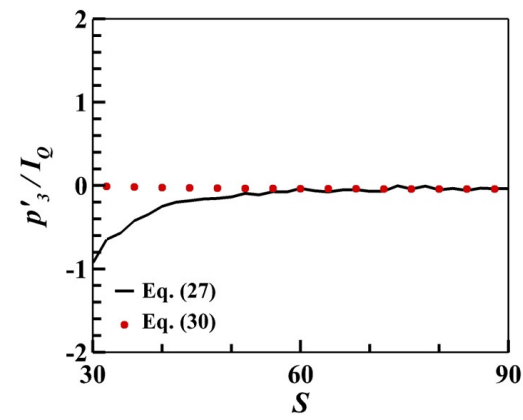

Figure 5 Sound pressures correspond to (a) the second term and (b) the third term at different observer distances $S$. The sound pressures corresponds to the second term are computed by using the Eqs. (26) and (29), respectively. The sound pressures corresponds to the third term are computed by using the Eqs. (27) and (30), respectively. The sound pressures at the non-time dimensional time $t=120$ are plotted. The convecting Mach number of the vortex is $M a=0.3$ and the observers are distributed along the line with $\theta=0^{\circ}$. 
(a)

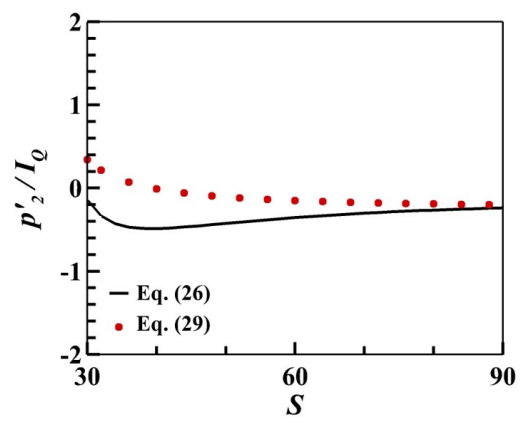

(b)

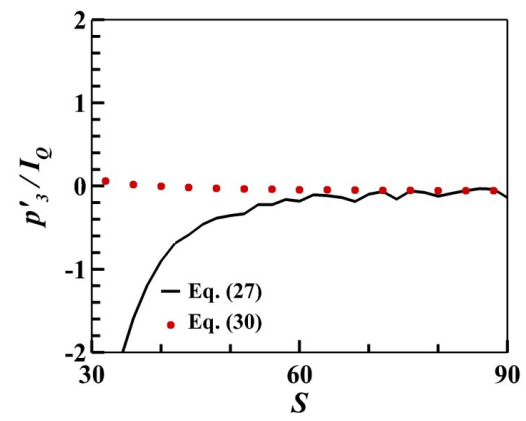

Figure 6 Sound pressures correspond to (a) the second term and (b) the third term at different observer distances $S$. The sound pressures corresponds to the second term are computed by using the Eqs. (26) and (29), respectively. The sound pressures corresponds to the third term are computed by using the Eqs. (27) and (30), respectively. The sound pressures at the non-time dimensional time $t=120$ are plotted. The convecting Mach number of the vortex is $M a=0.4$ and the observers are distributed along the line with $\theta=0^{\circ}$.

(a)

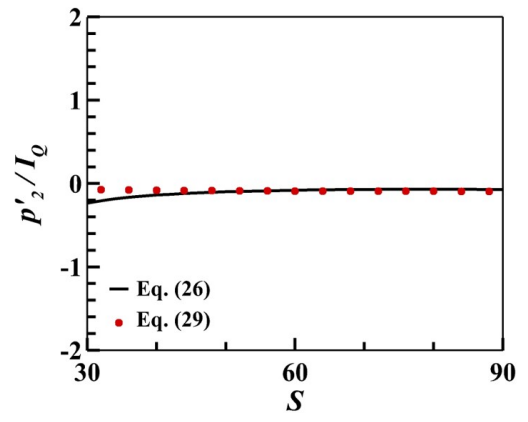

(b)

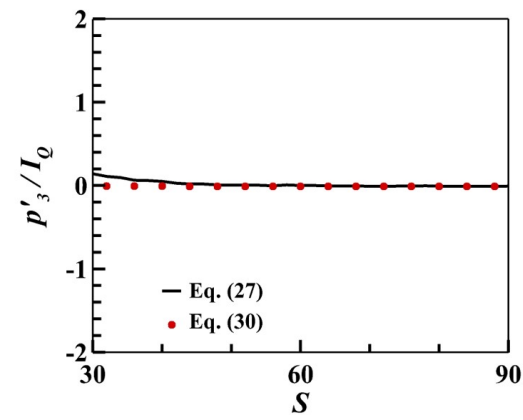

Figure 7 Sound pressures correspond to (a) the second term and (b) the third term at different observer distances $S$. The sound pressures corresponds to the second term are computed by using the Eqs. (26) and (29), respectively. The sound pressures corresponds to the third term are computed by using the Eqs. (27) and (30), respectively. The sound pressures at the non-time dimensional time $t=120$ are plotted. The convecting Mach number of the vortex is $M a=0.2$ and the observers are distributed along the line with $\theta=45^{\circ}$. 
(a)

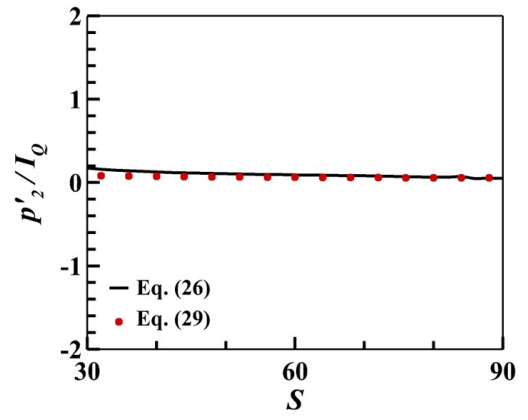

(b)

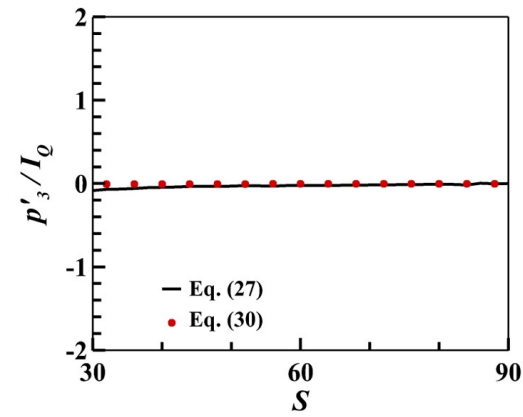

Figure 8 Sound pressures correspond to (a) the second term and (b) the third term at different observer distances $S$. The sound pressures corresponds to the second term are computed by using the Eqs. (26) and (29), respectively. The sound pressures corresponds to the third term are computed by using the Eqs. (27) and (30), respectively. The sound pressures at the non-time dimensional time $t=120$ are plotted. The convecting Mach number of the vortex is $M a=0.2$ and the observers are distributed along the line with $\theta=90^{\circ}$.

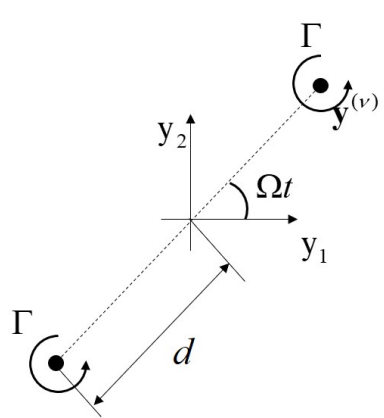

Figure 9 Schematics of a co-rotating vortex pair.

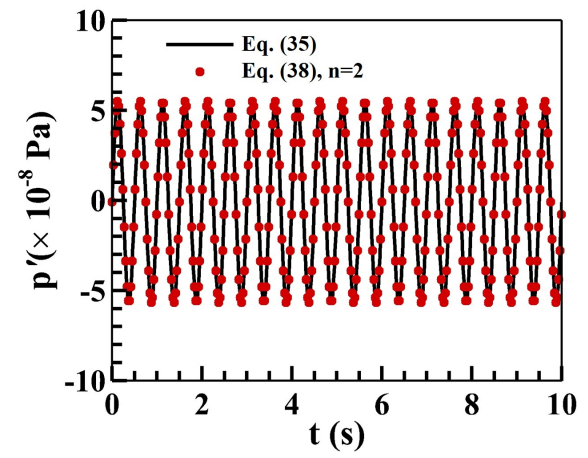

Figure 10 Acoustic pressure computed by using the acoustic pressure formulation (Eq. (35)) and the approximated acoustic pressure formulation (Eq. (38)). 


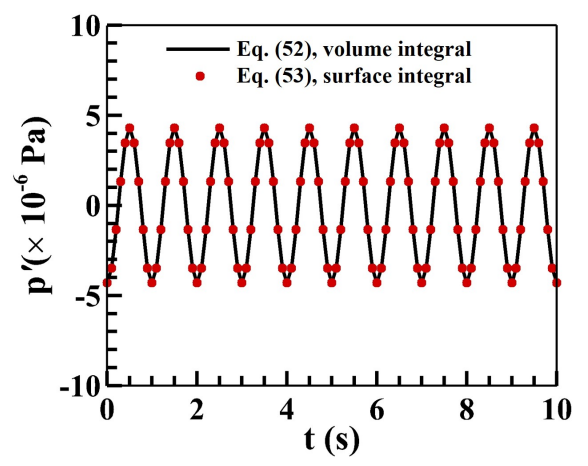

Figure 11 Contribution to far-field acoustic pressure from the homogeneous vortical flow computed by using (a) the volume integral (Eq. (52)) and (b) the surface integral (Eq. (53)). 